\title{
Brillouin Light Scattering from Surface Phonons in Hexagonal and Cubic Boron Nitride Films
}

\author{
T. Wittkowski, P. Cortina, J. Jorzick, K. Jung, and B. Hillebrands \\ Fachbereich Physik und Schwerpunkt Materialwissenschaften, Universität Kaiserslautern, Erwin-Schrödinger-Str. 56, 67663 Kaiserslautern, \\ Germany
}

\begin{abstract}
Phase velocities of surface acoustic waves in several boron nitride films were investigated by Brillouin light scattering. In the case of films with predominantly hexagonal crystal structure, grown under conditions close to the nucleation threshold of cubic BN, four independent elastic constants have been determined from the dispersion of the Rayleigh and the first Sezawa mode. The large elastic anisotropy of up to $c_{11} / c_{33}=0.1$ is attributed to a pronounced texture with the $c$-axes of the crystallites parallel to the film plane. In the case of cubic BN films the dispersion of the Rayleigh wave provides evidence for the existence of a more compliant layer at the substrate-film interface. The observed broadening of the Rayleigh mode is identified to be caused by the film morphology.
\end{abstract}

\section{Introduction}

The boron nitride and the carbon system are iso-electronic. Thus the physical properties of the hexagonal and cubic boron nitride modification, h-BN and c-BN, are very similar to those of graphite and diamond. This is why boron nitride, especially c-BN, became a material of wide scientific interest. Its hardness of about $70 \mathrm{GPa}$ is close to the outstanding hardness of diamond, but c-BN is superior to diamond in view of its thermal stability and its lower reactivity with ferrous materials like steel.

However, it is not only the hardness, but also properties like the friction coefficient and the ductility of the film material that are of decisive influence in technical applications. These parameters describe technologically relevant properties of the material, and they are correlated to the more fundamental stiffness tensor describing the elastic behavior. The mechanical properties of boron nitride coatings are strongly influenced by the deposition conditions. Since the theoretical prediction of the films elastic properties as a function of the deposition parameters is not possible up to now, the components of the stiffness tensor must be determined experimentally. This is the subject of this study. For this purpose the detection and analysis of surface acoustic waves in h-BN films and, for the first time, in c-BN films are presented.

\section{Film preparation}

The hexagonal films were grown in the group of Prof. F. Richter, TU Chemnitz. A detailed description of the apparatus and the sputtering process is given in [1]. Further investigations of the plasma processes involved, using optical emission spectroscopy (OES) and a Langmuir double probe technique, are reported in [2]. Here, the deposition parameters of two films, which were deposited onto the (100) surface of silicon, will be briefly reviewed. One film was grown by reactive d.c. magnetron sputtering from a heated boron target, and a second film by r.f. magnetron sputtering from a polycrystalline h-BN target. In both cases the distance between the target and the substrate was near $100 \mathrm{~mm}$. d.c. sputtering took place in an argon-nitrogen atmosphere of $0.2 \mathrm{~Pa}$ and an $\mathrm{Ar} / \mathrm{N}_{2}$ ratio of $9 / 1$. The discharge was sustained by a magnetron target power of 200 $\mathrm{W}$, the substrate biasing voltage was $-250 \mathrm{~V}$. The second film was r.f. sputtered under an atmosphere of $0.2 \mathrm{~Pa}$, a magnetron target power of $1000 \mathrm{~W}$ and a substrate bias of $120 \mathrm{~V}$. At the beginning of the deposition process the $\mathrm{Ar} / \mathrm{N}_{2}$ ratio of $97 / 3$ was kept for two minutes followed by sputtering in a pure nitrogen atmosphere. The two film thicknesses as determined by profilometry are $150 \pm 7 \mathrm{~nm}$ and $75 \pm 7 \mathrm{~nm}$, respectively. An analysis of the film composition in view of its crystallographic phases was made using FTIR transmission spectroscopy. For both films transmission minima were observed at $780 \mathrm{~cm}^{-1}$ and 1380 $\mathrm{cm}^{-1}$, corresponding to the out-of-plane and in-plane vibration of $\mathrm{sp}^{2}$-bonded $\mathrm{BN}$ [3]. A presence of $\mathrm{sp}^{3}$-bonds could not be detected. The deposition conditions were chosen to be close to the nucleation threshold but the threshold was not exceeded, probably due to a too low ion flux rate.

Cubic BN films were grown in the group of Prof. A. Lunk, Universität Stuttgart, in a hollow cathode arc evaporation device [4]. Two films were deposited onto (111) silicon with identical deposition parameters. The substrate temperature was $450{ }^{\circ} \mathrm{C}$, the $\mathrm{Ar} / \mathrm{N}_{2}$ ratio was $7 / 3$ at a total pressure of $0.6 \mathrm{~Pa}$. The anode crucible contained the boron material, which has an electrical conductivity high enough to sustain the discharge at elevated temperatures. Typical discharge powers are a few $\mathrm{kW}$, the substrate bias was -700 V. An analysis using transmission FTIR showed a layered film composition with a lower h-BN layer and an upper c$\mathrm{BN}$ layer [5]. In a least squares fit to the measured spectrum the film thicknesses were determined with high accuracy $( \pm 2 \%)$ [6], yielding $7.5 \mathrm{~nm}(6.5 \mathrm{~nm}) \mathrm{h}-\mathrm{BN}$ and a total thickness of $123 \mathrm{~nm}(277 \mathrm{~nm})$ for the thinner (thicker) film.

A third c-BN film was deposited under similar conditions. Its growth was controlled with in situ IR reflection spectroscopy [6]. The h-BN interface layer has a thickness of $30 \pm 10 \mathrm{~nm}$ and the total film thickness is $600 \pm 30 \mathrm{~nm}$.

\section{Experimental setup}

The elastic properties, i.e., the components $c_{\mathrm{ij}}$ of the stiffness tensor, are determined using Brillouin light scattering (BLS). This is an inelastic light scattering techniques which, as the primary experimental quantity, yields the dispersion of the 
frequencies of acoustic phonons as a function of the wavevector.

Light of the $514.5 \mathrm{~nm}$ line of an $\mathrm{Ar}^{+}$-laser is focused onto the sample. The scattered light is frequency analyzed by a Fabry-Pérot interferometer of Sandercock type in backscattering geometry $[7,8]$. A more detailed description of the BLS technique is given in $[8,9]$. The incident as well as the detected scattered light is polarized parallel to the scattering plane ( $p-p$ polarization). In backscattering geometry, wave vector conservation implies, that surface phonons with wavevector $q_{\|}$are detected, if

$$
q_{\|}=2 k \sin \theta \text {. }
$$

Here $k=|\vec{k}|$ is the absolute value of the wavevector of the incoming and reflected laser light, and $\theta$ is the angle of light incidence measured against the surface normal. Due to energy conservation the frequency shift $\Delta v$ of the backscattered light is

$$
\Delta \mathrm{v}=\frac{\mathrm{v}}{\pi} k \sin \theta,
$$

where $\mathrm{v}$ is the phase velocity of the surface phonon. Thus the dispersion of surface waves is examined by variation of the angle $\theta$ and the measurement of the frequency shift. The angle of incidence was varied from $\sin \theta=0.3$ to $\sin \theta=$ 0.95. Typical laser powers were in the range from $100 \mathrm{~mW}$ to $300 \mathrm{~mW}$.

\section{Results and discussion}

\section{1 h-BN films}

For both h-BN films most of the BLS spectra, recorded under various angles of incidence, showed two intensity maxima originating from inelastic phonon scattering. The [001]-direction of the silicon substrate was chosen to be the direction of surface wave propagation. Due to the angular dependence of the frequency shift the modes detected were identified to be the Rayleigh mode and a transverse guided mode (Sezawa mode) in the discrete part of the spectrum. Figure 1 shows the dispersion of the phase velocity, which depends on the dimensionless product of the surface phonon wavevector $q_{\|}$and the film thickness $h$ [10]. The calculated dispersion curves are obtained in a least squares fit to the experimental data with the elastic constants as free parameters using the model described in [10]. The fit results are summarized in Tab. 1. The film material is expected to possess the symmetry of the hexagonal crystals with the zonal axis fixed parallel to the surface normal. Hence, the medium is isotropic for wave propagation parallel to the surface, the surface normal acts as symmetry axis. Nonzero components of the tensor of the elastic constants are $c_{11}=c_{22}, \quad c_{33}, \quad c_{44}=c_{55}, \quad c_{66}=\left(c_{11}-c_{12}\right) / 2, \quad c_{12}$, and $c_{13}=c_{23}$. Since the solutions of the equation of motion for sagittal and horizontal shear waves decouple in the present case, the elastic constant $c_{12}$ is not needed to describe the sagittally polarized modes and we are left with the four independent elastic constants $c_{11}, c_{33}, c_{55}$, and $c_{13}$, which can be determined from the experiment.

As described in Sect. 2 both samples are grown under conditions close to the nucleation threshold of c-BN. Under these conditions, mainly characterized by a high ion flux and energy, volume diffusion takes place in the upper atomic layers of the film. The material grows in a dense $\mathrm{sp}^{2}$-bonded nanocrystalline network with a very low void density. Since the films do not contain $\mathrm{sp}^{3}$-bonded $\mathrm{BN}$, the mass density of single crystal h-BN, $\rho=2.27 \mathrm{~g} / \mathrm{cm}^{3}$, was used for the calculation.

As is seen in Tab. 1 the elastic properties of the d.c. sputtered and the r.f. sputtered films are rather similar. The shear modulus $c_{44}$ is about $45 \mathrm{GPa}$ for both samples. Because the shear modulus is related to the hardness [11], this is evidence for a rather hard material in view of plastic deformation.

Solutions of the equation of motion, which describe sagittally polarized modes in a film with axial symmetry, contain two terms, $A_{1}$ and $A_{2}$, which give rise for an elastic anisotropy. The first one is defined as the ratio of the extensional stiffness constants parallel and perpendicular to the surface,

$$
A_{1}=c_{11} / c_{33} \text {. }
$$

The second one is analogous to the anisotropy in the cubic system,

$$
A_{2}=2 c_{44} /\left(c_{11}-c_{13}\right) \text {. }
$$

$A_{1}=A_{2}=1$ is a condition sufficient for elastic isotropy
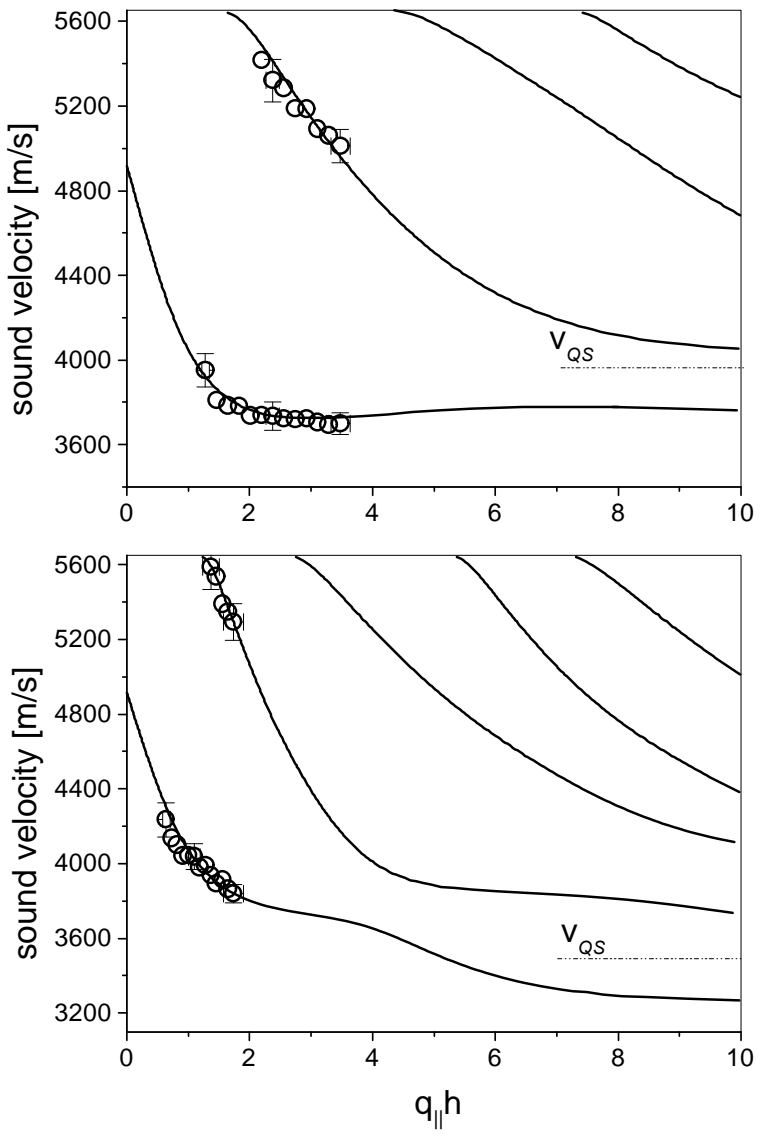

Fig. 1: Measured (open circles ) and fitted (lines) sound velocities of the BN film modes versus the product of wavevector $q_{\|}$and film thickness $h$. The upper and lower diagram show the dispersion of the r.f. sputtered film $(h=$ $75 \mathrm{~nm})$ and of the d.c. sputtered film $(h=150 \mathrm{~nm})$, respectively. Vertical error bars are due to the finite accuracy of the frequency determination in the BLS spectra, horizontal error bars are due to the finite accuracy in the determination of the film thickness. The phonon wavevector is varied by the angle of light incidence. The curves show the results of a least squares fit to the experimental data with the elastic constants as free parameters. The dotted line indicates the velocity of the quasi-shear bulk mode $\mathrm{v}_{Q S}$, travelling parallel to the surface. 


\begin{tabular}{l|lllll} 
& $\boldsymbol{c}_{\mathbf{1 1}}[\mathrm{GPa}]$ & $\boldsymbol{c}_{\mathbf{1 3}}[\mathrm{GPa}]$ & $\boldsymbol{c}_{\mathbf{3 3}}[\mathrm{GPa}]$ & $\boldsymbol{c}_{\mathbf{5 5}}[\mathrm{GPa}]$ & $\boldsymbol{A}_{\mathbf{1}}$ \\
\hline r.f. sputtered, $75 \mathrm{~nm}$ & 44 & 50 & 293 & 35 & 0.15 \\
d.c. sputtered, $150 \mathrm{~nm}$ & 38 & 8 & 392 & 55 & 0.10 \\
\hline error [\%] & 5 & 34 & 10 & 11 &
\end{tabular}

Tab. 1: Elastic constants and elastic anisotropy $A_{1}=c_{11} / c_{33}$ of two sputtered h-BN films. The constants are obtained from a least squares fit to the experimental data (see Fig. 1). The errors are due to the statistical deviation of the measured data with respect to the calculated curve.

concerning sagittal modes. The $A_{2}$ term behaves very sensitive if the denominator is near zero, which is the case for the r.f. sputtered sample. Because $c_{13}$ could be determined only with low accuracy the discussion will be confined here on the $A_{1}$ term.

Both samples exhibit a remarkable anisotropy, $A_{1}=0.15$ for the r.f. and $A_{1}=0.1$ for the d.c. sputtered film. This result is explained if the material is considered to consist of nonisotropically oriented crystallites. Crystalline h-BN and crystalline graphite are very comparable concerning their elastic properties. The covalent $\mathrm{sp}^{2}$-bonds with a short bond length lead to an extremely high stiffness of the basal plane, whereas, due to the weak interplanar $\pi$-bonds, the compliance is very large in this direction. Thus for graphite the extensional stiffness constant in the direction of the $c$ axis is about 40 times smaller than in a perpendicular direction [12-15]. Because of its iso-electronic configuration the anisotropy of h-BN is comparably high [16]. Therefore the experimental findings are evidence that the $c$-axes of the crystallites are oriented more or less parallel to the film plane with random in-plane orientation. At the interface as well as at grain boundaries the film material is likely to be amorphous. This BN component behaves essentially isotropic, hence the averaging with the crystalline components again reduces the overall anisotropy in comparison to the well-ordered hexagonal lattice.

These results are in good agreement with the findings of investigations on the growth mechanisms of c-BN using intense ion plating. One usually finds that amorphous $\mathrm{sp}^{2}-$ bonded $\mathrm{BN}$ of a few $\mathrm{nm}$ thickness exists in the region near to the interface to the substrate. In this region nanocrystalline h-BN or sub-modifications with less regular stacking order grow under conditions which shall induce the phase transition to c-BN for subsequently deposited material [6, 17-20]. Such deposition parameters force the film material to build up a biaxial compressive stress. The Gibb's free energy in a h-BN crystallite is lowered if the $c$-axis orients parallel to the stress field $[17,21]$. The free energy is minimized, consequently the $c$-axis orientation depends on the distance from the interface, and it reflects the actual internal stress at each growth stage.

Deposition conditions to grow c-BN are such that the $c$-axes are oriented parallel to the surface when the phase transition starts. In the investigated samples the phase transition to c$\mathrm{BN}$ has not yet occurred, hence the $c$-axes of the h-BN crystallites at the surface do not necessarily lie completely parallel to the film.

The elastic constants obtained with the BLS technique average the elastic behavior over the film thickness. They are therefore effective elastic constants. Taking this and, additionally, a dependence of the texture as a function from the distance to the interface into account, it is obvious that the measured anisotropy must be less pronounced than the anisotropy of a single crystal. An additional indicator for the dependence of the texture on the distance to the interface is the stronger anisotropy $\left(A_{1}=0.1\right)$ of the thicker film in comparison to the thinner film $\left(A_{1}=0.15\right)$ [9].

In the context discussed here minor emphasis was spent on the growth mechanisms on the $\mu \mathrm{m}$ thickness scale. Although a columnar growth, as is described by several models for ion plating deposition [22, 23] would induce anisotropy, the measured anisotropy is so large that it can only be explained satisfactorily by the consideration of the crystallite orientation as discussed above.

Going back to the dispersion curves in Fig. 1, it is seen that for large values of $q_{\|} h$ the phase velocities of the Sezawa modes converge to the velocity $\mathrm{v}_{Q S}$, which corresponds to a quasi-shear wave of the bulk material propagating parallel to the surface [24]. This velocity is found to be $\mathrm{v}_{Q S}=3963 \mathrm{~m} / \mathrm{s}$ and $\mathrm{v}_{Q S}=3495 \mathrm{~m} / \mathrm{s}$ for the d.c. and the r.f. sputtered film, respectively. A comparison with the transverse sound velocity, $\mathrm{v}_{T}=\sqrt{c_{55} / \rho}=4922 \mathrm{~m} / \mathrm{s}$ (d.c.) and $\mathrm{v}_{T}=3927 \mathrm{~m} / \mathrm{s}$ (r.f.), shows that $\mathrm{v}_{Q S}$ is remarkably smaller than $\mathrm{v}_{T}$, namely $959 \mathrm{~m} / \mathrm{s}$ and $432 \mathrm{~m} / \mathrm{s}$ for the d.c. and the r.f. sputtered film, respectively.

Geometrical features of slowness surfaces are discussed in general by Musgrave [25]. According to his analysis of the quasi-shear wave solution, the geometry of the slowness curve is related to the validity of three inequalities. Two of the inequalities, namely

$$
\begin{aligned}
& \left(c_{13}+c_{44}\right)^{2}>\left(c_{11}-c_{44}\right)\left(c_{33}-c_{44}\right) \text { and } \\
& \left(c_{13}+c_{44}\right)^{2}>c_{33}\left(c_{11}-c_{44}\right)
\end{aligned}
$$

are fulfilled for both films investigated, leading to the shape of the slowness curve shown in Fig. 2. Following Ref. [25] there are only a few non-organic materials (beryllium for example) which exhibit a similar behavior. As is shown above, it is the anisotropy that is responsible for any deviation from a circular slowness curve. It is obvious that a low $c_{11} / c_{33}$ ratio crucially influences the anisotropic behavior

The intersection of the slowness surface of the quasi-shear wave with any meridian plane is shown in Fig. 2 for the r.f. sputtered film. It is seen that pure modes for which the direction of propagation and energy flux coincide, appear at angles $0^{\circ}, 62.5^{\circ}$ and $90^{\circ}$ (in the first quadrant) with the surface normal. The projection of the slowness curve on a direction of propagation parallel to the film yields the minimum phase velocity of the quasi-shear wave $\mathrm{v}_{O S}$, to which all higher surface modes converge with increasing wavevector.

\section{$4.2 \quad$ c-BN films}

For the $123 \mathrm{~nm}$ and $277 \mathrm{~nm}$ thick c-BN films, grown under identical conditions, a single surface mode was detected under various angles of light incidence. The dispersion of the Rayleigh mode observed in the thin film was measured 


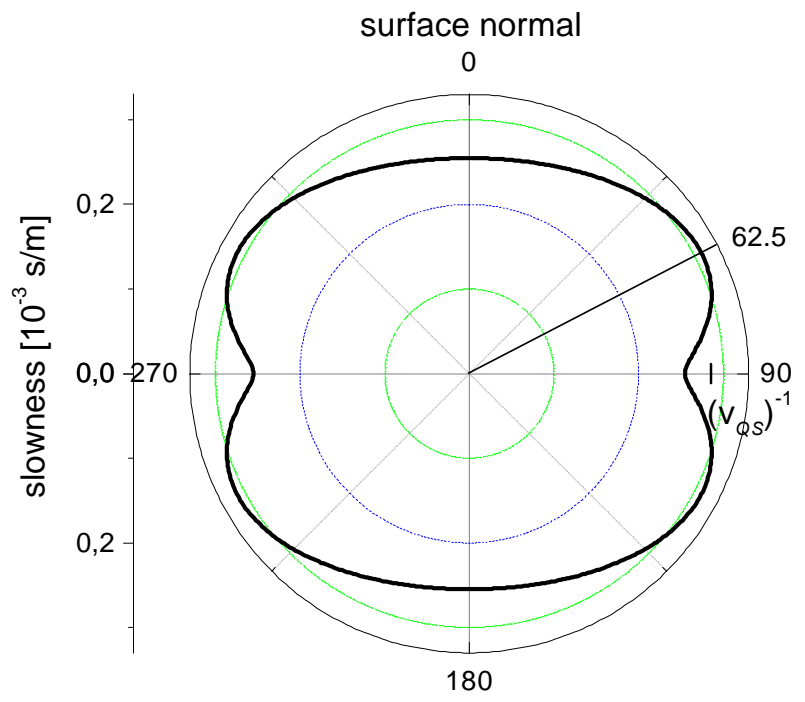

Fig. 2: Polar plot of the intersection of the slowness surface of the quasishear wave with any meridian plane for the r.f. sputtered sample. Pure modes propagate with the angles $0^{\circ}, 62.5^{\circ}$ and $90^{\circ}$ inclined to the surface normal. The projection of the curve on the direction of propagation parallel to the surface yields $\left(\mathrm{v}_{Q S}\right)^{-1}$. For large wavevectors the velocities of all Sezawa modes converge against the velocity limit $v_{Q S}$ (see Fig. 1).

up to values of $q_{\|} h$, at which the mode reaches the transverse threshold. For larger values of $q_{\|} h$ a leaky mode above the transverse threshold was detected. Its detection was limited to $\theta>40^{\circ}$ due to the increasing attenuation of the mode for smaller wavevectors. For large wavevectors the constant velocity of the film Rayleigh mode will be approached. Thus film and substrate constitute a system of type „fast film - slow substrate" with $\mathrm{v}_{\mathrm{T}, \text { film }} / \mathrm{v}_{\mathrm{T} \text {,substrate }}>\sqrt{2}$.

Figure 3 shows the dispersion behavior of the two films. The phase velocity of the thin sample at $q_{\|} h \approx 1$ lies remarkably below the Rayleigh velocity of the substrate, $\mathrm{v}_{R}$. Since the velocity must converge to $\mathrm{v}_{R}$ at $q_{\|} h=0$, the dispersion curve must have a pronounced minimum at $q_{\|} h$ between 0 and 1. Such a distinct deviation from the typical dispersion behavior of a substrate stiffening system can be understood only if a thin compliant interlayer at the substrate interface exists. Due to the lower sound velocity in this layer the Rayleigh velocity of the film is decreased below the Rayleigh velocity of the substrate for large wavelengths. In general, the existence of a dip in the dispersion curve cannot be excluded for some directions of propagation using a $\mathrm{Si}(111)$ substrate surface [26]. However, this effect is much too weak to describe the pronounced minimum in the measured dispersion curve. Therefore the existence of a more compliant interlayer must be concluded from the experiment. Evidence for a h-BN layer near the interface has already been obtained from the FTIR analysis.

The surface topography of the two samples was determined with an atomic force microscope (Topometrix). The topography of the surface as well as the corresponding BLS spectrum, taken under an angle of light incidence of $\theta=49^{\circ}$, is shown in Fig. 4. Compared to the $277 \mathrm{~nm}$ thick film the $123 \mathrm{~nm}$ thick film exhibits a remarkably rougher surface, smaller grains and consequently a higher number of internal boundaries than the thicker film. In the BLS spectra the disturbance of the propagation of the surface wave leads to an enhanced damping and thus to a larger line width of the Rayleigh mode. Since the displacement field of the

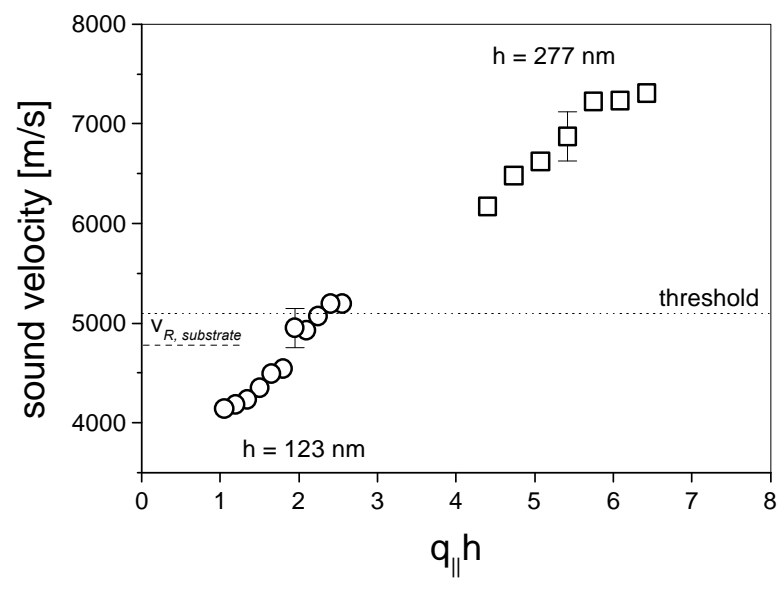

Fig. 3: Measured sound velocities of the surface modes of two c-BN films as in Fig. 1 versus the product of wavevector $q_{\|}$and film thickness $h$. The sound velocities of the thicker film (squares) lie far above the velocity threshold indicated by the dotted line. The sound velocities of the thinner film (circles) lie below the threshold and reach the threshold at $q_{11} h \approx 2.5$. The dashed line on the left indicates the Rayleigh velocity $\mathrm{v}_{R \text {,substrate }}$ of the (111) silicon substrate in [1 $1 \overline{1} 0$ ]-direction.

Rayleigh mode possesses a maximum amplitude close to the surface, the morphology of the film can be inferred from the line width.

This correlation seems to be surprisingly strong for the investigated films. One could expect the surface wave of the thinner sample to be less disturbed because of the stronger influence of the well ordered crystalline substrate. Also one could expect the surface wave of the thicker sample to be stronger damped because of the attenuation of this leaky mode. However, the experimental findings indicate that the film morphology has the dominating influence on the mode width.

BLS measurements on the third, $600 \mathrm{~nm}$ thick, c-BN film show the phase velocity of the Rayleigh mode to be independent of the phonon wavelength for this thickness. Because of the large film thickness the surface wave propagation is no more influenced by the lower h-BN layer $(30 \mathrm{~nm})$ and the substrate. From the slope of the straight line in Fig. 5 the Rayleigh velocity is found to be $\mathrm{v}_{R}=$ $7440 \pm 419 \mathrm{~m} / \mathrm{s}$.

In 1994 Grimsditch et al. determined the stiffness tensor of single crystalline c-BN with Brillouin light scattering [27]. The elastic constants $c_{11}=820 \mathrm{GPa}, c_{12}=190 \mathrm{GPa}$ and $c_{44}=$ $480 \mathrm{GPa}$ and the resulting bulk modulus $c_{\mathrm{B}}=400 \mathrm{GPa}$ agree fairly well with results of first principles calculations [28]. In order to compare the single crystal constants with the measurement in the present study, we need to take the texture of the investigated films into account. We apply the averaging processes developed by Voigt and Reuss [25]. The Voigt method implies a continuous strain across a crystallite boundary, and the Reuss method implies a continuous stress. Although both methods do not correctly take the elastic boundary conditions into account, they usually provide a good estimate of the effective elastic constants, in particular if the results of both methods are close to each other. These averaging procedures can be used for aggregates of arbitrarily oriented crystallites without a preferred orientation in the material, they leave the bulk 

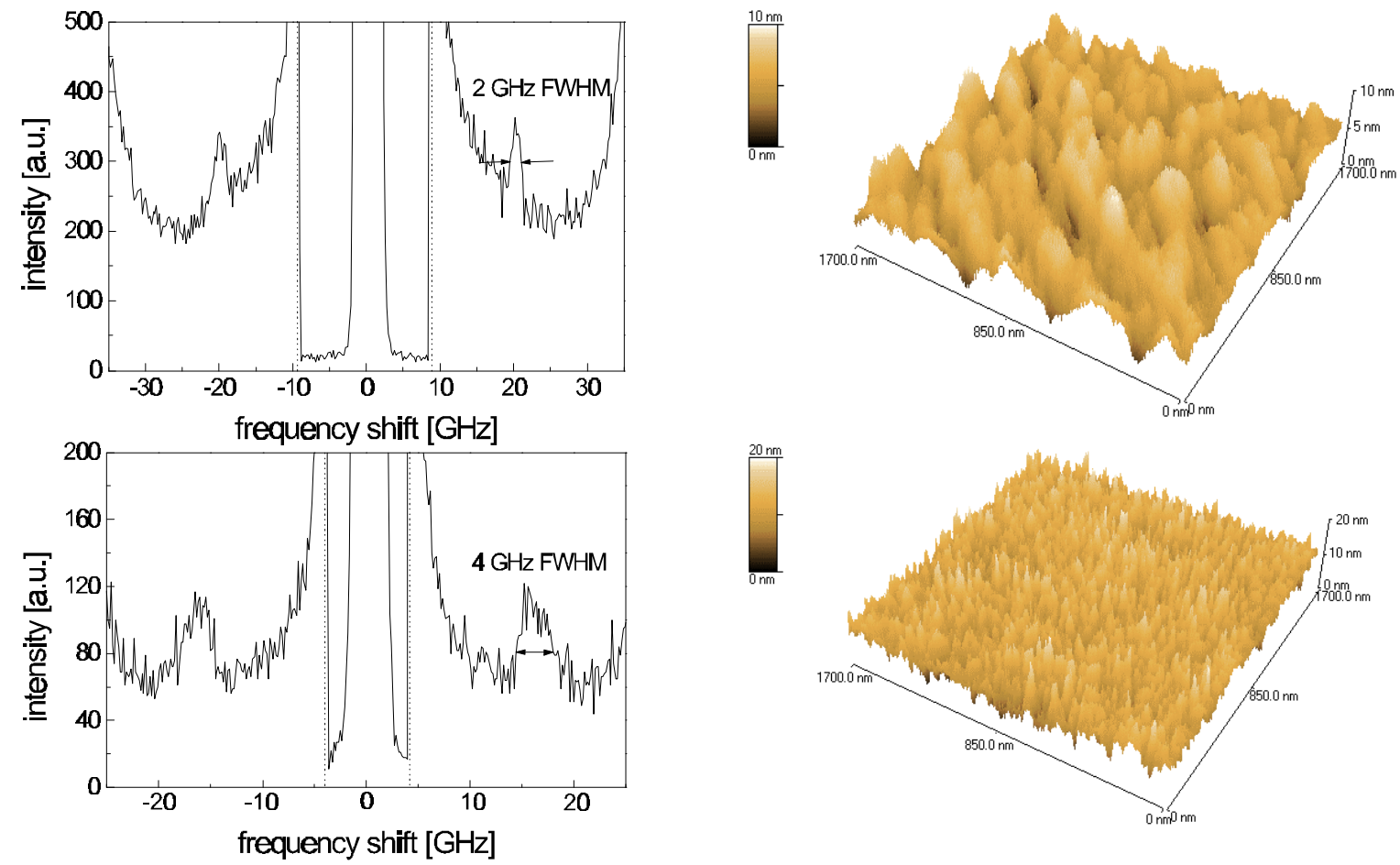

Fig. 4: Brillouin light scattering spectrum and surface topography of the $150 \mathrm{~nm}$ thick BN film (top) and the $75 \mathrm{~nm}$ thick h-BN film (bottom). The dotted lines in the spectra indicate the frequency range of the suppressed elastically scattered light. The energetic width of the Rayleigh mode, which is visible both on the Stokes and Anti-Stokes side of the spectra, correlates with the grain sizes at the surface of the samples.

modulus unchanged. The constants obtained by both methods, $c_{i j}^{\text {Voigt }}$ and $s_{i j}^{\text {Reuss }}$, are used in the empirical formula

$$
\bar{c}_{i j}=1 / 2 \cdot\left(c_{i j}^{\text {Voigt }}+\left[s_{i j}^{\text {Reuss }}\right]^{-1}\right),
$$

yielding the averaged set of elastic constants, $\bar{c}_{i j}$ [25]. These constants are $\bar{c}_{11}=941 \mathrm{GPa}, \bar{c}_{12}=130 \mathrm{GPa}$ and $\bar{c}_{44}=$ $401 \mathrm{GPa}$. The results of the Voigt and Reuss averaging, $c_{i j}^{\text {Voigt }}$ and $\left[s_{i j}^{\text {Reuss }}\right]^{-1}$, deviate between 1 and $4 \%$ from the averages $\bar{c}_{i j}$. The ratio between the Rayleigh velocity and the transverse sound velocity follows immediately to be $\mathrm{v}_{R} / \mathrm{v}_{T}=0.899$.

In order to calculate the shear modulus of the film material, $c_{44}=\mathrm{v}_{T}^{2} \cdot \rho=\left(v_{R} / 0.899\right)^{2} \cdot \rho$, the Rayleigh velocity

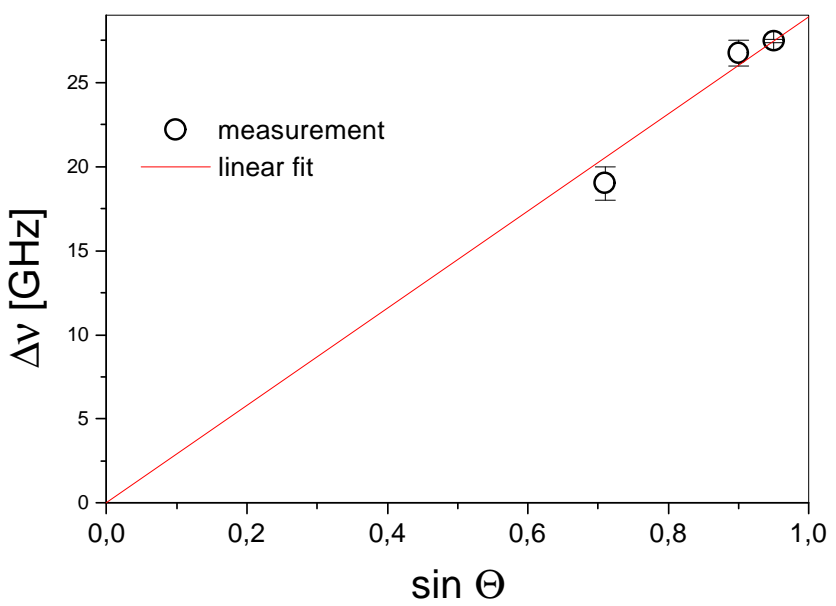

Fig. 5: Phonon frequency versus $\sin \theta$ for a $600 \mathrm{~nm}$ thick c-BN film. The sound velocity of the surface wave is not influenced by the material at the interface or the substrate due to the large film thickness. From the slope of the linear fit the Rayleigh mode sound velocity is $\mathrm{v}_{R}=7440 \pm 419 \mathrm{~m} / \mathrm{s}$. obtained in the BLS measurement is inserted in this equation. With the mass density $\rho=3.3 \mathrm{~g} / \mathrm{cm}^{3}$, which is about $95 \%$ of the single crystal density, the shear modulus of the c-BN film is $c_{44}=226 \pm 26 \mathrm{GPa}$. This value shows the film material to be very stiff, however, the discrepancy to the averaged single crystal constant $\bar{c}_{44}=401 \mathrm{GPa}$ is rather large. This deviation is attributed to the morphology of the analyzed film. The used averaging procedure does not take the crystallite and grain boundaries into account, which dominantly consist of $\mathrm{sp}^{2}$-bonded $\mathrm{BN}$ and whose consequences for the film elasticity are difficult to estimate quantitatively.

Measurements on a few hundred $\mu \mathrm{m}$ thick c-BN disks were performed with a dynamic resonance method by D'Evelyn et al. [29, 30]. For various samples the authors find shear moduli larger than $280 \mathrm{GPa}$ which mainly depend on the c$\mathrm{BN}$ content, the crystallite size and the sintering procedure. A comparison with the shear modulus derived in the present study shows the c-BN film to possess a lower rigidity. Possibly a high defect density of the c-BN crystallites in the film material is able to reduce the shear modulus additionally.

\section{Conclusion}

In conclusion we have shown that the elastic constants of $h$ $\mathrm{BN}$ films and c-BN films differ distinctly from the values of BN single crystals and sintered materials. The elastic behavior of these films, deposited with high ion fluxes, strongly depend on the deposition conditions. This demonstrates the importance to completely determine the elastic properties of $\mathrm{BN}$ films to be able to optimize their mechanical properties for technical applications. 


\section{Acknowledgements}

We would like to thank Dr. E. Weissmantel and Prof. Dr. F. Richter, TU Chemnitz, for the preparation of the h-BN samples and P. Scheible, L. Ulrich and Prof. Dr. A. Lunk, Universität Stuttgart, for supplying us with high quality cBN samples. Financial support by the Deutsche Forschungsgemeinschaft is gratefully acknowledged.

\section{References}

[1] R. Pintaske, Th. Welzel, N. Kahl, M. Schaller, J. Hahn, F. Richter, Surf. Coat. Technol. 90 (1997) 275.

[2] R. Pintaske, Th. Welzel, M. Schaller, N. Kahl, J. Hahn, F. Richter, Surf. Coat. Technol. 99 (1998) 266.

[3] R. Geick, C.H. Perry, G. Rupprecht, Phys. Rev. 146 (1966) 543.

[4] K.-L. Barth, A. Lunk, J. Ulmer, Surf. Coat. Technol. 92 (1997) 96.

[5] P.J. Gielisse, S.S. Mitra, J.N. Plendl, R.D. Griffis, L.C. Mansur, R. Marshall, E.A. Pascoe, Phys. Rev. 155 (1967) 1039.

[6] K.-L. Barth, W. Fukarek, H.-P. Maucher, M.F. Plass, A. Lunk, Thin Solid Films 313-314 (1998) 697.

[7] R. Mock, B. Hillebrands, J.R. Sandercock, J. Phys. E 20 (1987) 657.

[8] B. Hillebrands, Rev. Sci. Instrum. 70 (1999) 1589.

[9] T. Wittkowski, J. Jorzick, K. Jung, B. Hillebrands, Thin Solid Films 353 (1999) 137.

[10]G.W. Farnell, E.L. Adler, Physical Acoustics, W.P. Mason, R.N. Thurston (Eds.), Academic Press, New York, 1972, pp. 35-127.
[11]D.M. Teter, MRS Bulletin 23 (1998) 22.

[12] S.A. Lee, S.M. Lindsay, Phys. Stat. Sol. B 157 (1990) K83.

[13]J.F. Green, I.L. Spain, Phys. Rev. B 11 (1975) 3935.

[14]J.F. Green, T.K. Bolland, J.W. Bolland, J. Chem. Phys. 64 (1976) 656

[15]L. Ducleaux, B. Nysten, J.-P. Issi, A.W. Moore, Phys. Rev. B 46 (1992) 3362.

[16]R.W. Lynch, H.G. Drickamer, J. Chem. Phys. 44 (1965) 181.

[17]D.R. McKenzie, W.D. Fall, S. Reisch, B.W. James, I.S. Falconer, R.W. Boswell, H. Persing, A.J. Perry, A. Durandet, Surf. Coat. Techn. 78 (1996) 255.

[18]W. Donner, S. Chamera, A. Rühm, H. Dosch, S. Ulrich, H. Ehrhardt, Appl. Phys. A 65 (1997) 1.

[19]W. Dworschak, K. Jung, H. Ehrhardt, Thin Solid Films 254 (1995) 65.

[20]F. Richter, G. Krannich, J. Hahn, R. Pintaske, M. Friedrich, S. Schmidbauer, D.R.T. Zahn, Surf. Coat. Techn. 90 (1997) 178

[21]G.F. Cardinale, D.L. Medlin, P.B. Mirkarimi, K.F. McCarty, D.G. Howitt, J. Vac. Sci. Technol. A 15 (1997) 196.

[22]J.A. Thornton, J. Vac. Sci. Technol. 11 (1974) 666.

[23] R. Messier, A.P. Giri, R.A. Roy, J. Vac. Sci. Technol. A 2 (1984) 500.

[24]B.A. Auld, Acoustic Fields and Waves in Solids, Vol. 1, Wiley, New York, 1973.

[25] M.J.P. Musgrave, Crystal Acoustics, Holden-Day, San Francisco, 1970.

[26] G. Carlotti, D. Fioretto, L. Giovannini, L. Palmieri, G. Socino, L. Verdini, E. Verona, IEEE 1994 Ultrasonics Symposium, 457.

[27]M. Grimsditch, E.S. Zouboulis, J. Appl. Phys. 76 (1994) 832.

[28] K. Kim, W.R.L. Lambrecht, B. Segall, Phys. Rev. B 53 (1996) 16310.

[29] M.P. D‘Evelyn, K. Zgonc, Diam. Rel. Mat. 6 (1997) 812.

[30] M.P. D‘Evelyn, T. Taniguchi, Diam. Rel. Mat. 8 (1999) 1522. 